\title{
Influencing Factors on the Natural Frequency of Bending- Torsional Coupling Gear-Rotor
}

\author{
Yongqiang Che ${ }^{1,}$ a , Yue Han ${ }^{1}$, Junshan Guo ${ }^{1}$, Panfeng Shang ${ }^{1}$, Haizhen Lv ${ }^{2}$ \\ ${ }^{1}$ State Grid Shandong Electric Power Research Institute, Jinan 250002, China. \\ 2 Shandong Electric Power Industry and Pressure Vessel Inspection Center Co. Ltd, Jinan 250002, \\ China. \\ a cheyongqiang163@163.com
}

\begin{abstract}
Based on the finite element method, this paper establishes a differential equation of the coupled gear-rotor motion by applying MATLAB. The coupled bending and torsion vibration were considered in the equation. The eigenvalue and eigenvector were acquired through order reduction and solution of the homogeneous motion differential equation. The natural frequencies of bending vibration and torsional vibration were extracted. The influencing factors on the natural frequency of gear-rotor system were analyzed by changing gear meshing stiffness, gear transmission ratio and bearing stiffness, respectively. The results showed that the meshing stiffness of gears affected the natural frequency of torsional vibration, the bearing stiffness affected the natural frequency of bending vibration, and the gear transmission ratio did not affect the natural frequency.
\end{abstract}

Kay words: bending-torsional coupling, natural frequency, gear drive

\section{Introduction}

Because of reliable operation and constant transmission ratio, gear drive is the most widely applied transmission mechanism in rotary machinery. It can meet the requirement of high speed and high power transmission in modern industry [1]. The geared rotor system has been widely used and become an important type of transmission in the rotor system.

Both bending vibration and torsion vibration exist simultaneously in gear drive dual rotor system. Because of gear meshing between rotors, the bending and torsional vibrations of each rotor are coupled, and the vibration of each rotor is no longer independent, but affects and restricts each other. The gear, bearing and rotor have become a whole. So in the dynamic analysis of high speed geared rotating machinery, the gear meshing effect should be considered and the gear pair should be regarded as part of the rotor system [2].

Considering 5 degrees of freedom of bending and torsion vibration and using MATLAB in this paper, the motion differential equations of shaft unit, disk unit, gear pair unit and bearing unit were derived, then the overall motion differential equation of the gear-rotor was obtained by coupling. The eigenvalue and eigenvector were acquired through aligning the lower order and solution of the homogeneous motion differential equation. The natural frequencies of bending vibration and torsional vibration were extracted. The influence factors on the natural frequency of gear-rotor system were analyzed by changing gear meshing stiffness, gear transmission ratio and bearing stiffness respectively. 


\section{Geared Rotor Model}

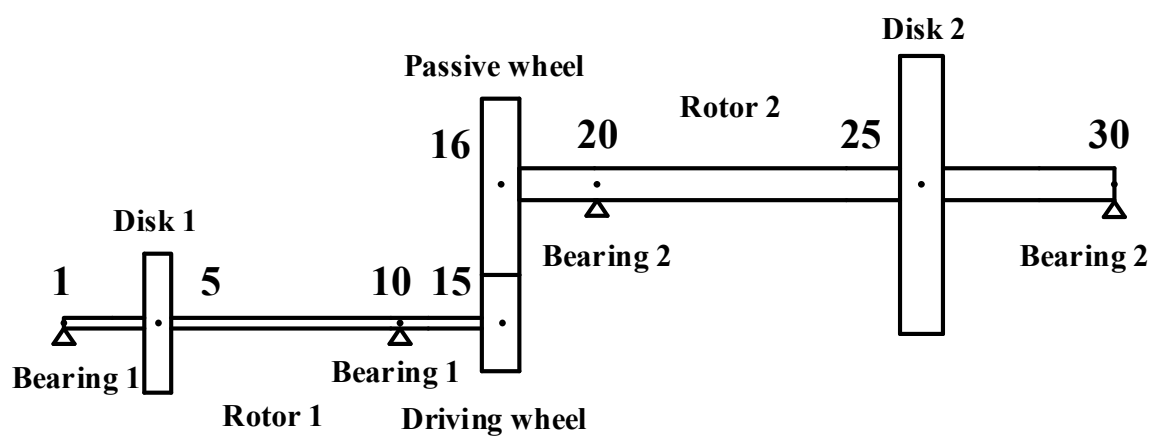

Fig.1 Schematic diagram of the structure of a turbo-generator system for a gear transmission

Fig. 1 is a schematic diagram of the structure of a turbo-generator system for a gear transmission, in which rotor 1 is the active rotor and rotor 2 is the passive rotor. Based on the finite element method of rotor dynamics, the model is discretized into bearing, shaft, disk, gear pair and other units, and a certain node is set on each unit. For the shaft unit, two nodes are set, located at two endpoints of the shaft unit. For the disk unit, one node is set at the center of the disk. For the bearing unit, one node is set at the center of the journal. Then, the motion differential equations of each unit are obtained by analyzing each unit. Finally, the motion differential equations of all units are combined by generalized coordinates.

Tab.1 Length and diameter of each axis section of the system

\begin{tabular}{c|c|c|c|c|c|c}
\hline No. & $1-5$ & $5-10$ & $10-15$ & $16-20$ & $20-25$ & $25-30$ \\
\hline Length $(\mathrm{m})$ & 0.2 & 4.0 & 0.4 & 0.2 & 5.0 & 0.3 \\
\hline Diameter(m) & 0.05 & 0.05 & 0.05 & 0.15 & 0.15 & 0.15 \\
\hline
\end{tabular}

Tab.2 Structural parameters of gear, disk and bearing

\begin{tabular}{c|c}
\hline Name & Value \\
\hline Disk 1 mass(kg) & 10.4 \\
\hline Disk 2 mass(kg) & 432.2 \\
\hline Passive wheel mass $(\mathrm{kg})$ & 730.6 \\
\hline Polar moment of inertia of disk $1\left(\mathrm{kgm}^{2}\right)$ & 0.3 \\
\hline Polar moment of inertia of disk $2\left(\mathrm{kgm}^{2}\right)$ & 20.74 \\
\hline Polar moment of inertia of driving wheel $\left(\mathrm{kgm}^{2}\right)$ & 0.02 \\
\hline Polar moment of inertia of passive wheel $\left(\mathrm{kgm}^{2}\right)$ & 115.72 \\
\hline Teeth of driving wheel & 38 \\
\hline Teeth of passive wheel & 228 \\
\hline Pressure angle of the dividing circle $\left({ }^{\circ}\right)$ & 20 \\
\hline Tooth width(m) & 0.1 \\
\hline Modulus(mm) & 9 \\
\hline Gear pair overlap & 1.6 \\
\hline Bearing 1 main rigidity $(\mathrm{N} / \mathrm{m})$ & $1.02 \times 10^{9}$ \\
\hline Bearing 2 main rigidity(N/m) & $1.84 \times 10^{9}$ \\
\hline Bearing 1 main damping(N/m) $(\mathrm{m})$ & 3000 \\
\hline Bearing 2 main damping(Ns/m) & 3000 \\
\hline
\end{tabular}

Considering the coupled flexural and torsional vibration of the system, each node has 2 translational motion, 2 bending and 1 torsion with a total of 5 degrees of freedom [3-5], then the generalized coordinates of this node can be expressed as:

$$
q=\left[x, y, \theta_{x}, \theta_{y}, \varphi\right]
$$


Where, the subscripts 1 and 2 respectively represent represent nodes 1 and 2 at both ends of the axial unit. $x$ and $y$ are the translational displacement, $\theta_{x}$ and $\theta_{y}$ are the corner, $\varphi$ is the axial twist angle.

Using Lagrange equation, the motion differential equation of each unit is obtained, and then

$$
\boldsymbol{M} \ddot{\boldsymbol{q}}+\boldsymbol{C} \dot{\boldsymbol{q}}+\boldsymbol{K} \boldsymbol{q}=\boldsymbol{F}
$$

Combined to obtain the motion differential equation of the gear-rotor system.

Where, $\boldsymbol{M}$ is the mass matrix, $\boldsymbol{C}$ is the damping matrix, $\boldsymbol{K}$ is the stiffness matrix, $\boldsymbol{F}$ is the generalized force matrix.

\section{Natural Frequency Factor Analysis}

The maximum amplitude wave peak of the unbalanced response of two rotors in gear transmission generally occurs at the natural frequencies of bending vibration and torsional vibration of each order of the gear-rotor system [6]. Therefore, it is of more practical significance to pay attention to the factors influencing the natural frequencies of bending vibration and torsional vibration of the gearrotor system.

\subsection{Influence of Gear Meshing Stiffness}

In order to analyze the influence of gear meshing stiffness on the natural frequency of the system, Campbell diagrams of the natural frequency of flexural vibration and the natural frequency of torsional vibration were respectively computed in this paper by changing the meshing stiffness from $10^{2}$ to $10^{16}$.

Seen from fig.2, the natural frequency of torsional vibration of the system increases with the increase of meshing rigidity of gears. This indicates that the increase of the meshing stiffness of gears improves the overall torsional rigidity of the gear-rotor system, and the natural frequency of the torsional vibration of the system increases accordingly. The natural frequency of the first order torsional vibration increases the most, indicating that the natural frequency of the first order torsional vibration of the system is the most sensitive to meshing stiffness. However, when the meshing stiffness increases to $10^{8}$, the natural frequency of all torsional vibration will not change, and all of them will show a horizontal straight line. This is because the meshing stiffness of gears at this time is much higher than the stiffness of the system itself, and the influence of the meshing stiffness of gears on the system has been stable. While with the change of meshing rigidity of gears, the natural frequency of system bending vibration has hardly changed. This indicates that the meshing stiffness of gears mainly affects the natural frequency of torsional vibration of the system, but not the natural frequency of bending vibration of the system.

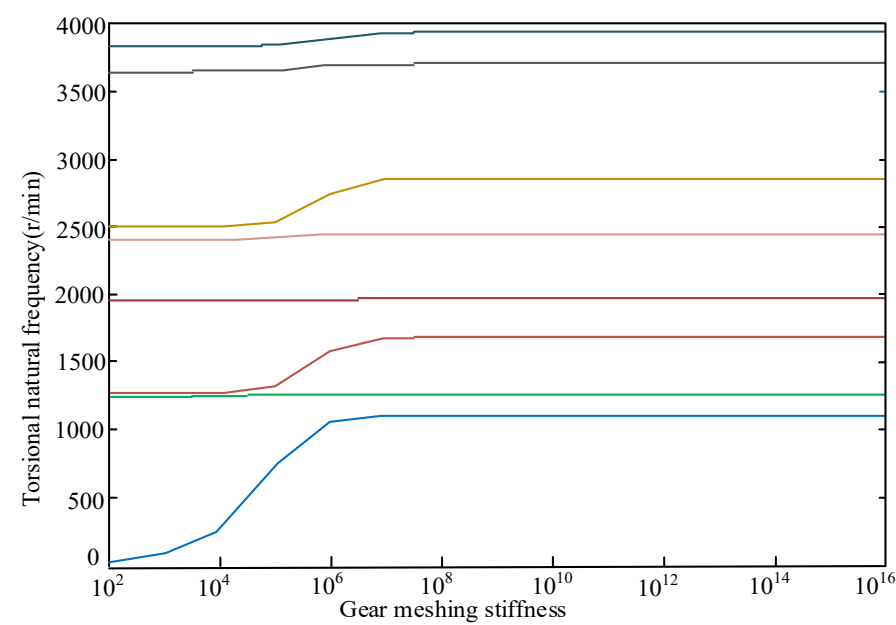

Fig.2 Natural frequency of torsional vibration -- meshing stiffness of gears 


\subsection{Influence of Gear Transmission Ratio}

Fig. 3 (a) and Fig. 3 (b) respectively show the change curves of bending natural frequency and torsional natural frequency with the increase of rotating speed when the transmission ratio is 1 and 10.Comparing the two figures, it can be found that the difference in gear transmission ratio does not affect the torsional vorticity line, the torsional vorticity line is always a horizontal straight line. However, the dynamic curves of some curved vortexes vary greatly, while the dynamic curves of other curved vortexes remain basically the same, especially when the transmission ratio is 10 , the positive vortexes deviate more from the reverse vortexes.

The change of gear transmission ratio actually changes the working speed of driven rotor 2 , thus changing the gyro effect of rotor 2 , which has a greater influence on rotor 2 . The rotation speed and gyro effect of rotor 1 did not change. Therefore, in Fig. 3 (a) and Fig. 3 (b), the unvarying curved vortexes should correspond to rotor 1 , and rotor 2 should correspond to the greater deviation of the positive and negative vortexes.

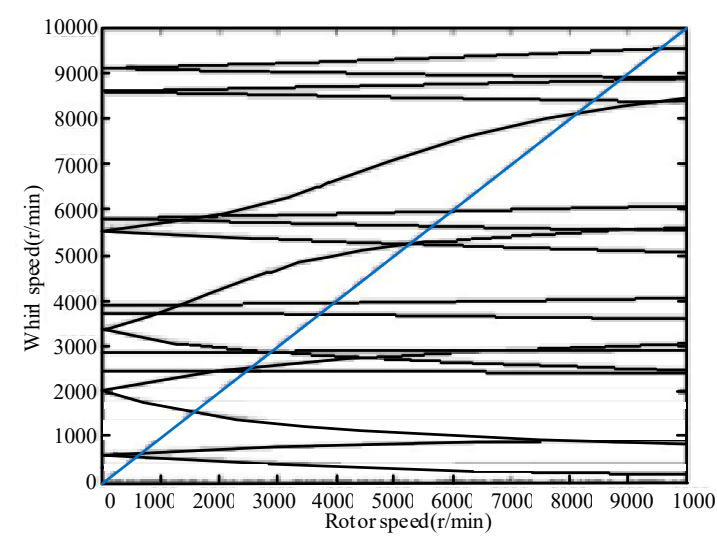

Fig.3(a) Transmission ratio is 1

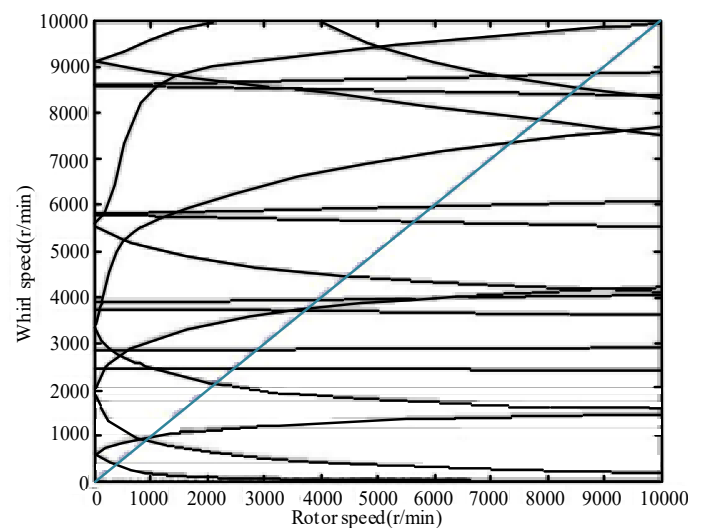

Fig.3(a) Transmission ratio is 10

Fig. 3 Campbell diagram with different transmission ratios

When the speed of active rotor 1 is 0 , the change of gear transmission ratio has no influence on the speed of rotor 2 , and it will not cause the change of the natural frequency of the system. Therefore, in order to analyze the influence of the transmission ratio, it is assumed that the rotation speed of rotor 1 is $5000 \mathrm{r} / \mathrm{min}$. At this speed, by changing the transmission ratio of gear from $1 / 6$ to 10 , the variation diagram of the first 5 order synchronous forward and backward vorticity frequencies of rotor 2 is obtained, as shown in Fig. 4. Seen from Fig. 4, with the increase of transmission ratio, the deviation between synchronous forward vortex frequency line of rotor 2 and synchronous reverse vortex frequency line is getting larger and larger. Therefore, the increase of gear transmission ratio not only improves the natural frequency of synchronous forward vortexes, but also reduces the natural frequency of synchronous reverse vortexes.

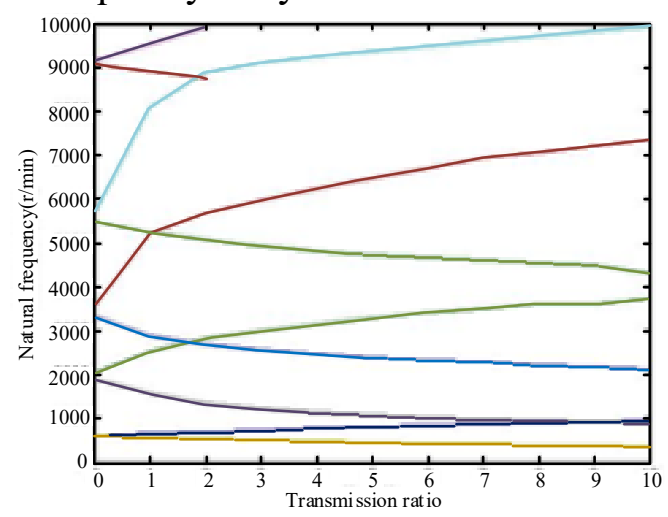

Fig. 4 First 5 order vortex frequency -transmission ratio of rotor 2

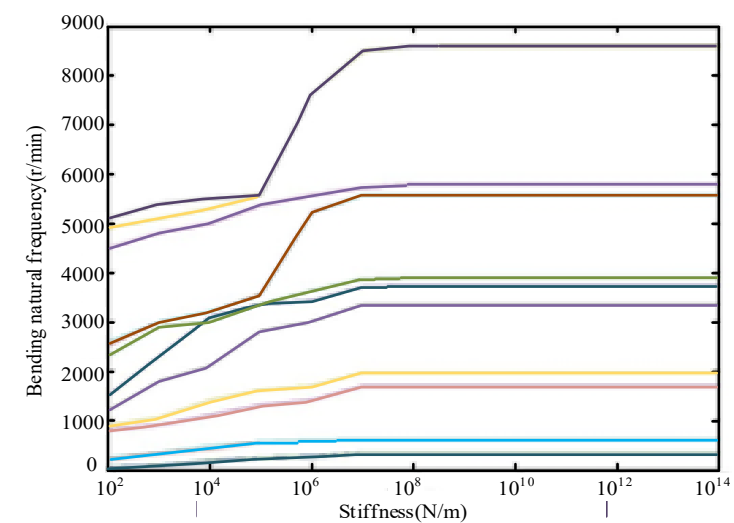

Fig.5 Natural frequency of bending vibration -bearing stiffness 


\subsection{Influence of Bearing Stiffness}

To facilitate analysis, the stiffness of the four bearings in the gear-rotor system model established in this paper is of the same order of magnitude, all of which are $10^{9}$. Campbell diagrams of bending natural frequency and torsional natural frequency were obtained respectively by changing the bearing stiffness from $10^{2}$ to $10^{14}$. Seen from Fig. 5, when the bearing stiffness is $10^{2}-10^{8}$, the bending natural frequency of the system increases with the increase of bearing stiffness. This is because the stiffness of the bearing increases, which increases the stiffness of the whole system of the gear-rotor. However, when the bearing stiffness increased to $10^{9}$, the natural frequency of bending vibration of the system did not change and tended to be stable. This is because the stiffness of the bearing is greater than that of the rotor itself, and the bending natural frequency of the system is stable under this order of magnitude. The natural frequency of torsional vibration of the system does not change with the increase of bearing rigidity, because the change of bearing rigidity will not cause the change of system torsional rigidity.

\section{4 effects of Other Gear Parameters}

The change of gear parameters affects the meshing stiffness of gear. In this paper, it was found that changing gear's tooth number, modulus, pressure angle, coincidence degree and thickness under the condition of unchanged transmission ratio would cause the change of gear meshing stiffness. However, the meshing stiffness of the gear-rotor system model established in this paper was at an order of magnitude of $10^{9}$, it could be concluded from the analysis in 3.1 that the natural frequency of bending vibration and torsional vibration are relatively stable at this order of magnitude and will not change much. Therefore, it could be considered that the change of gear's tooth number, modulus, pressure angle, coincidence degree and thickness had little influence on the natural frequency of the gear-rotor system.

\section{Conclusion}

In this paper, the model of bending and torsional vibration of geared rotor was established, and the influence of gear meshing stiffness, gear ratio, bearing stiffness and other gear parameters on the natural frequency of bending and torsional vibration were analyzed by calculation. The natural frequency of torsional vibration could be improved by increasing the meshing stiffness of gear, especially the natural frequency of the first order torsional vibration; but it had no effect on the natural frequency of bending vibration. The increasing transmission ratio had great influence on the bending vibration of active rotor, which could improve the natural frequency of synchronous forward vortex and reduce the natural frequency of synchronous reverse vortex; but it had no influence on the torsional natural frequency of the whole system and the bending natural frequency of passive rotor. The increase of bearing stiffness could improve the bending natural frequency of the whole system, but had no effect on the torsional natural frequency of the whole system. Under the condition of a constant transmission ratio, it could be considered that the change of gear's tooth number, modulus, pressure angle, coincidence degree and thickness had little influence on the natural frequency of the gear-rotor system.

\section{References}

[1]. LI Liangjun. Mechanical design. Higher Education Press, 2010.

[2]. LI Guoping. Study on vibration characteristics of gear - bearing - rotor system. Northeastern University, 2015.

[3]. CHE Yongqiang, XU Jingxia, QIAN Xiaodong, et al. Coupled bending and torsional vibrations of geared rotor system. Mechanical and electrical engineering. 2012, 29 (6): 632-635,649. 
[4]. JIANG Qinglei, WU Dazhuan, TAN Shanguang, et al. Development and application of a model for coupling geared rotors system. Journal of vibration engineering. 2010,23(3): 254-259.

[5]. ZHONG Yi'e. Rotor dynamics. Tsinghua university press, 1987.

[6]. HAN Yuqiang, SHI Shouhong, ZHANG Suohuai. Influence of gear coupling on the inherent characteristics of rotor-bearing system. Mechanical science and technology. 2003, 22 (1): 66-70. 